\title{
ISOGAL and 2MASS Study of the Central Regions of Our Galaxy
}

D. K. Ojha

T.I.F.R., Homi Bhabha Road, Colaba, Mumbai - 400 005, India

\author{
A. Omont, S. Ganesh, and ISOGAL Team \\ Institut d'Astrophysique de Paris, CNRS, Bd. Arago, F-75014, Paris
}

\begin{abstract}
We report the study of ISOGAL fields in the outer galactic bulge $\left(-1.5^{\circ}<\ell<+1.6^{\circ},-2.6^{\circ}<b<+6.0^{\circ}\right.$, area $\left.\sim 0.42 \mathrm{deg}^{2}\right)$. We have combined $15 \mu m$ and $7 \mu m$ ISOCAM observations with 2MASS $\mathrm{JHK}_{s}$ data to determine the nature of the sources and the interstellar extinction. Most of the ISOGAL sources show evolution of mass-loss rates in the range $3 \times 10^{-8}$ to $1 \times 10^{-6}\left(\mathrm{M}_{\odot} /\right.$ year $)$. Most of the detected sources are red giants above the RGB tip; a few of them show an excess in $\mathrm{J}_{-} \mathrm{K}_{s}$ and $\mathrm{K}_{s}$-[15] colors. These sources are AGB stars with large mass-loss rates.
\end{abstract}

\section{ISOGAL and 2MASS observations}

ISOCAM (Cesarsky et al. 1996) observations at $6^{\prime \prime}$ resolution of 12 bulge fields were used in this paper (Ojha et al. 2000, in preparation). These observations were performed during 1996-1998 at $15 \mu m$ (filter LW3 : 12-18 $\mu m$ \& LW9 : 14$16 \mu \mathrm{m}$ ), and at $7 \mu \mathrm{m}$ (filter LW2 : 5.5-8.5 $\mu \mathrm{m} \&$ LW6 : 7.0-8.5 $\mu \mathrm{m}$ ). In order to ensure a reasonable level of reliability, completeness and photometric accuracy, we presently limit the discussion of ISOGAL data to sources brighter than 8.5 mag $(8 \mathrm{mJy})$ for $15 \mu \mathrm{m}$ sources and $9.75 \mathrm{mag}(11 \mathrm{mJy})$ for $7 \mu \mathrm{m}$ sources. The near- infrared data used in this paper were acquired from 2MASS survey in the three bands, $\mathrm{J}(1.25 \mu \mathrm{m}), \mathrm{H}(1.65 \mu \mathrm{m})$ and $\mathrm{K}_{s}(2.17 \mu \mathrm{m})$. The scientific results of the analysis of other ISOGAL fields are described in Pérault et al. (1996), Omont et al. (1999) and Glass et al. (1999). The cross-correlation between ISOGAL and 2MASS sources has been made, which provide the multicolor data. These cross-correlated data allow us to discuss the nature and properties of individual sources, as discussed in the paper (Ojha et al. 2000, in preparation). The adjunction of 2MASS near-infrared data adds much to ISOGAL data, by providing different and more sensitive color indices, as well as estimates of the interstellar reddening.

\section{The nature of ISOGAL sources and mass-loss}

We have studied various magnitude-color \& color-color diagrams of ISOGAL/2MASs sources. In these diagrams, the ISOGAL sources form a mass-loss sequence start- 
ing with RGB tip stars having low to intermediate mass-loss rates and rising up to high mass-losing AGB stars. The mass-loss rates for the ISOGAL sources are calculated using a grid of models by Martin Groenewegen (private communication) for circumstellar envelopes. AGB stars with high mass-loss rates $\left(1 \times 10^{-6}\right.$ $\mathrm{M}_{\odot} /$ year) comprise $\sim 2 \%$ of the sources, with most of the remaining sources being low and intermediate mass-loss rate $\left(3 \times 10^{-7} \mathrm{M}_{\odot} /\right.$ year $)$ AGB stars.

The brightest stars detected at mid-infrared wavelengths form a loose group in the various color-magnitude diagrams. They are brighter than the tip of the sequence of "intermediate-AGB" sources. These stars are inside a box defined by Omont et al. (1999) for AGB stars showing high mass-loss rates. The bolometric magnitude for these sources peaks around $\mathrm{M}_{b o l} \sim-4$. These stars have larger mass-loss rate $\left(\geq 3 \times 10^{-6} \mathrm{M}_{\odot} /\right.$ year $)$ than for the intermediate-AGB sequence, which marks the onset of the superwind. Glass et al. (1999) identify them as miras which show lower mass-loss rates than $\mathrm{OH} / \mathrm{IR}$ stars.

\section{Conclusion}

We have shown that the combination of near- infrared (2MASS) and mid- infrared ( 7 and $15 \mu \mathrm{m}$ ) ISOGAL data allows reliable detection of AGB stars above the RGB tip. We conclude that most of the ISOGAL sources detected both at 7 and $15 \mu \mathrm{m}$ in the bulge fields are intermediate AGB stars or RGB tip stars with low and high mass-loss rates. The sequence in various color-magnitude diagrams coincides well with the late M AGB sequence, from M6 to M9, just above the RGB tip. The most important conclusion from the present analysis is that the ISOGAL sources detected in the bulge show the evolution of mass-loss rates in the range : $3 \times 10^{-8}$ to $1 \times 10^{-6}\left(\mathrm{M}_{\odot} /\right.$ year $)$.

\section{Acknowledgments}

This publication makes use of data based on observations with ISO, an ESA project with instruments funded by ESA Member States (especially the PI countries : France, Germany, the Netherlands and the United Kingdom) and with the participation of ISAS and NASA. This publication also makes use of data products from the 2MASS Survey, which is a joint project of the University of Massachusetts and the Infrared Processing and Analysis Center.

\section{References}

Cesarsky, C., et al. 1996, A\&A, 315L, 32

Glass, I.S., et al. 1999, MNRAS, 308, 127

Omont, A., et al. 1999, A\&A, 348, 755

Pérault M., et al. 1996, A\&A, 315, L165 